\title{
Recommendations on: internal standard criteria, stability, incurred sample reanalysis and recent 483s by the Global CRO Council for Bioanalysis
}

\author{
The Global CRO Council (GCC) for Bioanalysis was formed in an effort to bring together many CRO leaders to \\ openly discuss bioanalysis and the regulatory challenges unique to the outsourcing industry"
}

Steve Lowes', Jim Jersey ${ }^{2}$, Ronald Shoup ${ }^{3}$, Fabio Garofolo ${ }^{\dagger 4}$, Natasha Savoie ${ }^{4}$, Ejvind Mortz ${ }^{5}$, Shane Needham ${ }^{6}$, Maria Cruz Caturla ${ }^{7}$, Ray Steffen $^{8}$, Curtis Sheldon', Roger Hayes ${ }^{10}$, Tim Samuels", Lorella Di Donato' ${ }^{12}$, John Kamerud ${ }^{13}$, Steve Michael'4, Zhongping (John) Lin ${ }^{15}$, Jim Hillier ${ }^{16}$, Marc Moussallie ${ }^{17}$, Leonardo de Souza Teixeira ${ }^{18}$, Mario Rocci Jr'19, Mike Buonarati ${ }^{20}$, James Truog ${ }^{21}$, Saleh Hussain ${ }^{22}$, Richard Lundberg ${ }^{23}$, Alan Breau ${ }^{24}$, Tianyi Zhang ${ }^{24}$, Jianine Jonker ${ }^{25}$, Neil Berger ${ }^{26}$, Sofi Gagnon-Carignan ${ }^{27}$, Corey Nehls ${ }^{28}$, Robert Nicholson ${ }^{29}$, Martijn Hilhorst ${ }^{30}$, Shane Karnik ${ }^{31}$, Theo de Boer ${ }^{32}$, Richard Houghton ${ }^{33}$, Kirk Smith ${ }^{34}$, Laura Cojocaru ${ }^{35}$, Mike Allen ${ }^{36}$, Tammy Harter ${ }^{37}$, Saadya Fatmi ${ }^{38}$, Farhad Sayyarpour ${ }^{39}$, Jenifer Vija ${ }^{40}$, Michele Malone ${ }^{41}$, Dennis Heller ${ }^{42}$

Note that due to equality principals of Global CRO Council for Bioanalysis, authors are presented in alphabetical order of company name

${ }^{\dagger}$ Author for correspondence: Algorithme Pharma Inc., 575 Armand-Frappier Blvd, Laval (Montreal) Quebec, H7V 4B3, Canada Tel.: +| 450973 6077; Fax: +| 450973 2446; E-mail: fgarofolo@algopharm.com

Author affiliations can be found at the end of this article

Keywords: bioanalysis $₫$ bioanalytical CRO - bioanalytical guidelines « bioanalytical harmonization » bioanalytical issues $\approx \mathrm{GCC} \approx$ Global CRO Council

The 2nd Global CRO Council (GCC) for Bioanalysis Closed Forum was held on 15 April, 2011 in Montreal, Canada. In attendance were 45 senior-level representatives from 39 CROs on behalf of eight countries. The meeting began with the following admonition statement: "Our discussions today are subject to the anti-trust guidance applicable in Canada. Nothing discussed at this meeting is intended to restrict the individual decision-making of any participating company or to represent an agreement to coordinate marketing or sales conduct. Those participating in this meeting are instructed to avoid discussion of competitively sensitive subjects, including confidential marketing, sales and pricing information". Once this statement was acknowledged and accepted by all participants, the GCC's mission and vision were reviewed. The vision of the GCC is that CROs will have a greater influence if they speak with one voice on current topics of regulated bioanalysis. In order to accomplish this vision, a mission statement was presented and accepted by all members, stating that the GCC will focus on the discussion of regulatory developments, communicate their recommendations to international regulatory agencies that are open to discussion, communicate with other bioanalytical organizations (e.g., the Calibration and Validation Group [CVG], the European Bioanalysis Forum [EBF] and the American Association of Pharmaceutical Scientists [AAPS]) and provide input to the recently formed Global Bioanalytical Consortium (GBC).

Some achievements have already been noted since the Council's inception. First, the summary article from the 1st Closed Forum [1] has been very well received by the research community and it is currently one of the most read articles of Bioanalysis [101]. Second, the Boston Society - Applied Pharmaceutical Analysis (BSAT-APA) has followed the example of the $\mathrm{EBF}$ and announced that they are allowing CRO

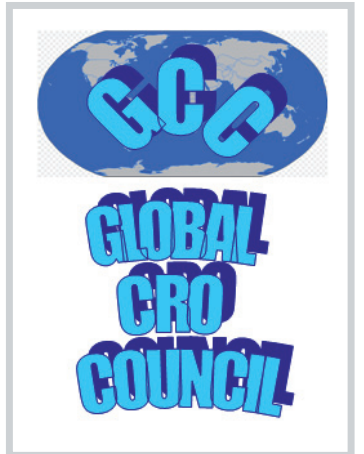

嫼 
representatives to participate in committees and the organization of programing [2]. Finally, the GCC has already expanded since September 2010 with the addition of 11 new member companies, mainly from Europe.

\section{'GCC is unique!'}

During the 2nd Closed Forum, it was reiterated that the GCC is unique since it is the companies that are members rather than individuals and each is equal within the organization (i.e., there is no hierarchical structure). In an effort to accommodate the busy schedules of the CRO representatives, GCC meetings will continue to be tied to major conferences where attendance by member companies is anticipated. Due to the global nature of this Council, it was agreed that additional closed forums should also be held in Europe and/or Asia.

A proposal was presented to formalize the Council under the umbrella of another nonprofit, scientific organization. A survey was conducted to determine if members were interested in pursuing this approach. Results indicated that a vast majority of members found that the current structure (closed forum, admonition statement and equal participation) as described in the first GCC article [1] was more than adequate and no further action was needed.

Feedback on the GCC was presented by old and new members indicating that reaction to the Council has been well received by clients and regulatory agencies. There was an initial concern that clients might not approve of competing companies meeting in such a manner. However, given that the group focuses on scientific issues, acts with transparency and quickly disseminates meeting minutes in the form of published reports, no concern has been raised to date about the value of the GCC. Furthermore, by sharing experiences and findings, agencies and clients anticipate that these forums will ensure that similar issues are rapidly addressed by all industry providers without multiple citations and new agency policies will be quickly and more efficiently propagated, thereby reducing the regulatory burden for all. CROs are unique in that they work with many different pharmaceutical companies and agencies, which results in a unique and comprehensive perspective on scientific approaches in relation to regulatory requirements.

At the 1st Closed Forum, it was decided that the Council would communicate via surveys because of their efficiency and speed. For example, the meeting agenda for the 2 nd Closed
Forum was developed by using a survey sent to all member companies with a list of proposed topics for discussion. TABLE $\|$ has the results of the survey, which indicate that the top five topics of the agenda would be:

- Internal standard (IS) variability and criteria;

- Stability issues: definition of 'fresh' and lightsensitivity;

- Incurred sample reanalysis (ISR);

- Whole blood stability and recent EBF recommendations;

- Sharing recent 483 s and other regulatory findings.

\section{Recommendation no. I: IS variability \& criteria}

The following general requirements for selection of an IS were acknowledged by all the members: the chosen compound should structurally resemble the analyte of interest (structural analogue or stable label) such that it behaves similarly during sample preparation and analysis. The IS that is added to each sample compensates for unavoidable assay variance due to, for example, extraction efficiency, ionization effects and transfer losses, and is highly recommended for LC-MS/MS assays. A good stable labeled IS that perfectly tracks the analyte (e.g., ${ }^{13} \mathrm{C}$ IS) improves both precision and accuracy of the assay.

It was concluded that IS variability does not necessarily predict assay reliability. A highly variable IS response may still be part of a method that is delivering accurate and precise concentrations whereas a narrow range of IS responses can still be associated with an assay that has fundamental problems. Thus, pre-determined IS criteria alone may not always be appropriate. Scientists/project leaders should review a method and evaluate the IS response variability using sound scientific judgment.

Based upon the discussion, two approaches for defining criteria for IS variability were discussed. The first approach is to set an upper and a lower boundary for IS response (e.g. 50-150\% of the mean IS response of known samples) that triggers a response or action should the criteria fail to be met. The second approach is to perform a trend analysis: to use the IS variation of known samples to define the acceptability of the IS variation for unknown samples.

Both approaches have their advantages and disadvantages. Traditionally, clients and agencies 
Table 1. Survey results for topics to discuss at the 2 nd Global CRO Council for Bioanalysis closed forum.

Topic

Sharing recent 483 /findings

Internal standard variability and criteria

Whole blood stability and recent European Bioanalysis Forum recommendations

Stability issues: definition of 'fresh', sample collection, light-sensitive, impact of hemolysis on stability

Incurred sample reanalysis

Method qualification versus validation for nonclinical biomarker analyses

Hemolysis: matrix effect and stability - how to quantify hemolysis in real incurred samples

Ligand-binding assay: method and lot comparison studies

Harmonization for assay transfer

Validation of dried blood sample methods

Re-injection vs reanalysis vs nonreportable values

Incorporation of Global CRO Council for Bioanalysis

Quality management system: application of aspects of ICH Q7A and Q10 to bioanalysis

Global multicenter studies: blank matrices and crossvalidation considerations

How to create consistency among inspectors

Agency efforts to amend the good laboratory practices and the opportune time to move towards a more

global consensus

Matrix stability assessments in ligand-binding assays

Implications to acceptable changes to mobile phase, injection volume, flow rate, column temperature and good

manufacturing process approach

Elemental analysis (e.g. ICP-MS): what and what not to validate

Society of Quality Assurance Annual Meeting: update on bioanalytical harmonization by BASS, BQSI, GBC

Importance to bring the old method to the current standard for supporting ongoing studies

Evaluation of lipemic plasma

prefer using a numerical criterion. In this manner, the assessment of IS variation is clear: the result is acceptable or it is not. This approach is easy to train and easy to use. The upper and lower limits are typically selected to identify samples that may have undergone an analytical error (e.g., missing or double addition of IS). Other than taking this reason into account, the selection of a numerical criterion is largely arbitrary. There is no scientific rationale for setting one range for all methods and analytes.

The use of trend analysis is highly recommended for all types of data analysis and can be applied to IS response evaluations. For example, using the variability of IS response of known samples to evaluate the unknown study samples is a better scientific approach than using a simple numerical rule, but this can be overly subjective and lapses in consistent interpretation do occur. The difficulty presented with this approach would be in the production of a standard operating procedure (SOP), which would promote a consistent and sound interpretation since the application is batch dependent and would require adequately trained scientists in its practice.

Several case studies were used to illustrate the benefits of this approach. In the first example, the results of a batch of approximately 200 samples were sorted based on IS area response. In this format, it was easy to determine that the IS responses of acceptable known samples (standards and quality control samples) were spread throughout the batch and sufficiently bracketed the unknown study samples. The spread of the low to high IS responses for the known samples was $18 \%$, and any non-bracketed unknown sample IS response was required to be within $18 \%$ of the range of the IS responses of the known samples. If not, the sample was investigated. In this way, the criterion set for the batch is not an 'acceptance' criterion, but rather a criterion to trigger an investigation.

A second example demonstrated the dangers of using a blanket criterion (50-150\%) for all batches. A batch of $>200$ samples was evaluated using the same process described above. Despite the fact that the IS responses of the unknown study samples were within the arbitrary $\pm 50 \%$ criteria, more than half of the unknown study samples had lower IS responses than the known samples (based on the spread, as mentioned previously). Since the samples failed the trend analysis, an investigation was necessary in order to prove that the IS compensated for any matrix
No. of votes

20

14

11

11

11

10

7

7

6

3

3

3

2

2

2

2

1

1

1 1

1

1 
and recovery effects. This case demonstrates that setting a numerical value can exclude situations that warrant an investigation.

Some past regulatory observations were discussed in order to better understand regulator perspectives on this topic. The first was a case where the agency thought inappropriate a prespecified criterion used (unspecified) because it failed to identify apparently low IS responses of some samples. An all too common occurrence is for industry to react to these citations by changing the numerical value to ensure that it meets the agency expectations. The Council recommended that the industry consider the context of the citation to the fullest extent possible before readjusting any numerical rules. Before such an extreme reaction, laboratories should instead consider initiating an investigation specifically aimed at the run or method in question.

To further expand this point, a second regulatory observation was presented that involved study samples that met the predefined IS criteria, but the laboratory applied its scientific judgment and decided that the results were anomalous. The study samples were investigated, the original data was found to be inaccurate and the study samples were repeated. However, a finding was issued because the company failed to follow its own criteria, even though it was ultimately proven that it was correct to investigate the study samples. After an in-depth discussion, dialogue within groups such as the GCC, other cross-industry consortia and, hopefully ultimately, with the governing agencies, can mediate and appropriately address the evolution of bioanalytical criteria. Clearly evolution of, and regulating against, simplified criteria can be inconsistent with sound scientific practice.

The GCC recommendation for the evaluation of IS response is shown in Box $\|$.

\section{Recommendation no. 2: stability issues}

Several articles have recently been published providing valuable insight into bioanalytical sample stability [3,4,102]. However, there are still issues that arise during inspections related to stability that seem to indicate that further discussions and clarifications are needed for both industry and regulatory agencies.

The first topic was in relation to the definition of freshly spiked standards/QCs hereby referred to as 'fresh'. Regulatory inspectors have cited that stability samples were not compared with fresh calibration standards. The Council clarified that, in general, agencies view 'fresh' as same day, 'within 24 h' or 'same calendar day'. However, it was agreed that for large molecule assays, even $24 \mathrm{~h}$ is often too long a duration to consider an analyte in matrix as fresh.

Furthermore, it was discussed if there was a need to define if calibration standards should be freshly extracted or freshly spiked, and if the latter, then whether freshly weighed and prepared stock solutions are also required. The consensus by the GCC was that freshly weighed and prepared stock solutions do not add value. Stock solutions that are used within the time period documented as being stable are defendable.

Furthermore, freshly spiked standards are required for matrix stability evaluations, and these samples should be used within a reasonable and appropriate time after spiking. A matrix sample that has been frozen is not fresh, but a sample that has been proven to be stable for several hours is considered 'fresh'. This is a recognized dilemma however, because in order to demonstrate that samples are stable over the short term, freshly spiked standards are required, but time must be allowed to prepare the fresh standards necessary to demonstrate preliminary stability data. Thus it becomes necessary to identify when 'time

\section{Box 1. The GCC recommendation for the evaluation of internal standard response.}

- During method development, the selection of the internal standard (IS) should be such that it will adequately track the analyte throughout sample analysis. An IS is added to samples to compensate for unavoidable sample losses (e.g., extraction transfer losses and ionization effects) and so variability is expected. A highly variable IS may not always be an indication of an uncontrolled analytical process. In some instances, it can be used to identify suspected sample manipulation errors such as double IS additions during sample preparation. Known samples within an analytical run should be used to scientifically evaluate the impact of the variability of the IS responses. Fluctuations in the IS response across samples are normal and acceptable; fluctuations across the responses of unknown samples should be similar to the fluctuations across the responses of known samples (especially for a stable label IS), with outliers noted and investigated as necessary. The criteria based on the fluctuation of known samples should be applied to the unknowns, taking into account the limits of the assay at the lower and upper end of the dynamic range. The criteria for IS response variation should be used to trigger an investigation and not for batch acceptance. Any numerical criteria should be data-driven based on method performance instead of a blanket criterion. However, investigations require time and money, and balance is needed between time/resources and providing quality results. To this end, one may choose to use a combined approach, that is, apply IS trend analysis on top of a blanket criterion. 
zero' $\left(\mathrm{t}_{0}\right)$ occurs. These time constraints must be established by stability data, first from a working knowledge of stock and working solution data and then from benchtop stability of the analytes in matrix. This information should be prudently gleaned from method development experiments before the start of formal validation.

The GCC recommendation for the assessment of analyte stability is shown in Box 2 .

It was clarified during this discussion that although freshly spiked standards are required for stability evaluations, an exception should be made for post-extraction stability. A false impression has been propagated based on erroneous information given at the Crystal City II conference, where it was stated that freshly spiked standards are required when evaluating post-extraction stability [5]. Freshly spiked standards are not necessary for this evaluation but they must be freshly extracted [4,6,7].

Another stability issue that was discussed was the stability of light-sensitive compounds. An FDA observation was presented wherein study samples were not protected from light despite the fact that the certificate of analysis for the compound stated that it was light-sensitive. Upon review it became apparent that suppliers do not necessarily have the appropriate evidence to make such claims of (in)stability, and any such evidence may have been generated under forced light conditions, not ambient light conditions, and on a different state of the drug (i.e., powder vs solution, solution vs in matrix) than what is being used in the laboratory. Benchtop stability in matrix provides documented evidence that study samples treated in a similar fashion are also stable.

The GCC recommendation for the stability of light-sensitive compounds is shown in Box 3 .

\section{- GCC recommendation for stability of} light-sensitive compounds

In order to use the reference standard and process samples unprotected from light, stability will need to be demonstrated using comparison samples that have been protected from light. This evaluation should be done during method development, at a minimum. Alternatively, if the reference standard supplier has additional information regarding stability of the standard in light, the certificate of analysis can be updated with the appropriate documentation.

\section{Recommendation no. 3: ISR}

Much discussion has been generated since the FDA introduced the necessity of the ISR evaluation at the Crystal City III meeting [8]. Procedures have been implemented industry-wide and the GCC endorses the evaluation since ISR has shown major and unquestionable scientific merit so far. However, some issues unique to CROs have been observed during the implementation of ISR across the industry. The nature of the $\mathrm{CRO}$ is to provide a service for a sponsor; however in some cases, sponsors have been declining ISR evaluations as part of some of their studies. As an example, the sponsor may not want ISR testing included in pilot studies. A sponsor could also request that their SOP be used, which may not meet a regulatory agency's requirements for this evaluation. Nevertheless, regulatory agencies expect that their guidance is being followed by the CRO. But what if the sponsor's demands conflict with the regulatory agency guidance? How does the CRO approach this situation since the CRO does not own the study specimens?

The GCC recommendation on the necessity to perform ISR is shown in Box 4.

The assessment of ISR has brought forward a question regarding the necessity for determining incurred sample accuracy (ISA). The Council heard one member's rationale for such an experiment, including a proposed experimental design to test for ISA [8]. It was reasoned that the total error of the measured value of an incurred sample consists of the sum of random errors and systematic errors. ISR experiments are designed

\section{Box 2. The GCC recommendation for the assessment of analyte stability.}

Matrix is spiked using stable stock solutions and used immediately until reliable method development and/or benchtop stability data are available.

\section{Box 3. The GCC recommendation for the stability of light-sensitive compounds.}

- In order to use the reference standard and process samples unprotected from light, stability will need to be demonstrated using comparison samples that have been protected from light. This evaluation should be done during method development, at a minimum. Alternatively, if the reference standard supplier has additional information regarding stability of the standard in light, the certificate of analysis can be updated with the appropriate documentation. 
Box 4. The GCC recommendation on the necessity to perform incurred sample reanalysis.

- The typical approach has been to document the client's decision in the study data. If an agency issues a finding to the $\mathrm{CRO}$ despite this documentation, the $\mathrm{CRO}$ should request the wording of the finding be adjusted so that it is clear that the CRO informed the sponsor of the regulatory liability risk yet the sponsor declined. Thus, the responsibility lies with the sponsor. The GCC requests that this type of situation is addressed during the GLP modernization process in order to clarify the responsibility of the sponsor versus the CRO.

to test for random errors, but ISA would test for systematic errors. In general, the presented procedure required a standard addition to incurred samples. The difference in the value obtained using the standard addition of equal quantity of analyte and the original concentration would be an indicator of method accuracy (i.e., within $\pm 20 \%$ as for ISR of small molecules). Furthermore, analysis of the correlation (comparison of the correlation coefficient, slope and intercept) between spiked and nonspiked incurred samples would provide an indication of similarity.

The GCC recommendation on the necessity to determine ISA is shown in Box 5 .

\section{Recommendation no. 4: whole blood stability}

The pertinence of whole blood stability to plasma assays is discussed in the FDA Bioanalytical Method Validation guidance [9] and the Crystal City III conference report [10] by briefly stating the requirement to assess the stability of analytes during sample collection, handling and storage rather than issuing specific recommendations on the conduct of the experiment. Similarly, while the EMA Draft Guideline on Validation of Bioanalytical Methods includes a more specific statement stating that the method must demonstrate that the concentrations obtained "reflect the concentrations of the analyte in the subject at the moment of sampling" [11], neither document prescribes a method for performing this evaluation, and consequently the industry has adopted various approaches.

The approach most frequently used by the majority of GCC representatives in attendance is the validated plasma method. Specifically, sufficient aliquots of whole blood are fortified and equilibrated. Time zero $\left(t_{0}\right)$ samples are drawn and are then immediately centrifuged to harvest plasma. The remaining blood samples are maintained at the desired test condition(s), and then aliquots are drawn and centrifuged to harvest plasma. The plasma aliquots are analyzed simultaneously and compared to assess stability for the desired time period.

However, any partitioning of analyte into red blood cells, cell surface binding or platelet binding is not taken into account, thereby masking potential analyte instability. Nonetheless, most attendees felt that this method is similar to variations that would occur in the actual sample collection process, is simple to perform and uses a validated method to perform the evaluation. In instances where analyte binding to blood components is evident, alternative approaches must be developed.

The EBF recommended an alternative approach using a qualified whole blood method. This was presented at the 3rd Open Conference in Spain in December 2010. This approach involved fortifying whole blood samples and then analyzing aliquots of stability and comparison samples directly from whole blood using a qualified bioanalytical method such as protein precipitation. It has been agreed that this approach has scientific value since it alleviates issues due to partitioning and binding. However, concerns were raised over using a qualified method that may not be well controlled to perform the evaluation. Furthermore, it should be noted that this method is not suitable for large-molecule assays.

The GCC recommendation on whole blood stability evaluation is shown in Box 6 .

Box 5. The GCC recommendation on the necessity to determine incurred sample accuracy.

Although the incurred sample accuracy (ISA) approach proposed is scientifically valid, there was concern expressed about introducing errors or false assumptions. Regulatory agencies do not require ISA assessments because the ISR experiment is already used as a surrogate for accuracy. The GCC does not recommend systematically performing this evaluation unless there is a clear reason for performing it as part of an investigation. 


\section{Box 6. The GCC recommendation on whole blood stability evaluation.}

- The whole blood stability should first be performed by using a 'validated plasma method' (plan A), and the 'qualified whole blood method' approach (plan B) should be used only if the validated plasma method is not appropriate or further investigation into blood stability is required due to analyte binding to blood components or other factors.

\section{Recommendation no. 5: recent 483 findings}

Several recent FDA findings were shared and discussed on the subjects of system conditioning, stability of co-administered compounds, validation reporting, calibrant and QC preparation and carryover.

\section{- System conditioning}

It is accepted practice that some bioanalytical methods require conditioning of the chromatographic column with plasma extracts prior to the analysis of sample batches to obtain reproducibility. An observation was issued because written procedures that described LC-MS/MS system equilibration were not available. Moreover, the citation objected to the use of study samples for system equilibration. The study under review was rejected because the inspectors suggested that there was a potential for fraud given that unknown study samples were used to condition the system. The finding was predicated on the potential of being able to predetermine the validity of the result of the sample, thereby biasing the study results. Moreover, using an old curve to precondition the system was also not acceptable because there was the possibility of using the same curve for a subsequent batch after it was already proven to be acceptable.

- GCC recommendation on the use of samples for system conditioning

CROs should have an SOP in place that outlines the types of samples used for conditioning runs and performing system suitability. This SOP would also specify system suitability acceptance criteria. Any samples used should be clearly identified and never used for any purpose other than as conditioning samples. If previously run samples are used (unknowns, standards or QCs), they must be pooled so as to remove any link to reported results. System suitability samples and the conditioning process should be defined by the method.

Stability of co-administered compounds The issue of analyte stability in the presence of co-administered compounds has been under discussion within the industry for some time. Several observations have been issued by the FDA for lack of supporting analyte stability data in the presence of co-administered compounds. The Agency declared that the available long-term frozen storage stability data of the individual components were not sufficient to support the stability of study samples. Specifically, study samples contained several co-administered compounds, whereas the long-term samples did not contain the same compounds in combination. After an extensive discussion, the Council concluded that there has been no scientific evidence that would indicate that the long-term stability of any analyte is compromised by the presence of co-administered compounds.

The GCC proposal and recommendation on the stability of co-administered compounds is shown in BOX 7 AND TABLE 2.

\section{Validation reporting}

Another observation was issued pertaining to all rejected method validation evaluations not being presented in the validation report. The evaluations referenced had been performed under a different study number, and had thus not been included in the method validation report.

The GCC recommendation on rejected evaluations in validation reports is shown in Box 8.

\section{Calibrant \& QC preparation}

Another regulatory observation obtained by two member companies cited that calibrants and QC samples were spiked using the same lot of stock solution. This finding was directly opposed to decisions presented in the Crystal City III conference report [10], which stated that this practice was acceptable as long as solution stability and accuracy were previously verified. In both cases, stock solutions were used within their stability periods, and stock check procedures demonstrated that the weighings were accurate. Even so, the use of a single stock solution for both calibrants and QC samples was cited as unacceptable. From the information collected during the forum, it seems that the FDA no longer regards stock check procedures 


\section{Box 7. The GCC proposal \& recommendation on the stability of co-administered compounds.}

- It was proposed during this forum that a joint effort be organized between as many CROs as possible to then generate sufficient combination stability data to provide a proof-of-concept if co-administered drugs can have an influence on the stability of the drug in a biological matrix. The data could then be presented to the US FDA and other regulatory agencies to address recent citations on the need to show the stability in presence of co-administered drugs and limit the requirement for this experiment in future.

- The initiative was launched by Eric Woolf (Merck) and Surendra Bansal (Roche) and brought to the GCC's attention. This initiative was very well received by the GCC's representatives. A survey was distributed to all the members and the preliminary results from the $28 \mathrm{CROs}$ that had responded as of 27 May 2011, are listed in TABLE 2.

- Final data are currently being collected and further coordination of this effort is ongoing.

\section{Table 2. Preliminary GCC survey results for co-administered compounds stability data.}

\section{Qustion Answer}

Do any CRO laboratories have data from nonproprietary compound combinations that they are willing to share? It is understood that proprietary compound data are not something we can provide

If no specifics can be provided, can members inform how many such coadministered stability investigations have been performed in your laboratory?

Have you ever seen a case where there was an observed instability due to the coadministered drug?

Have you been inspected and cited on this issue?
Ten have stability data on non-proprietary compounds and are willing to share stability data. Nine have stability data on only proprietary compounds. Eight have no stability data on coadministered compounds

Stability data are available on more than 103 nonproprietary compounds (some for as long as 2 years) and 60 proprietary compounds

Only one case is presently under investigation due to failing benchtop stability results, however, this has not been attributed to the presence of coadministered compounds

Seven CROs have stated that they have been cited by a regulatory agency on this issue as sufficient to adequately demonstrate accuracy, and a precision and accuracy batch is now required. However, it was unclear if this precision and accuracy batch was required in order to prove the accuracy of each stock weighed prior to use, or if only one precision and accuracy batch was required during method validation to confirm that only one stock was required for future use of the method.

The GCC recommendation on stock solutions used for calibrants \& QC sample preparation is shown in Box 9.

\section{Carryover}

Controlling carryover is always a challenge in bioanalytical methods. An observation was issued because the Agency did not agree that carryover had been controlled when the company injected its batches using a sequence ordered based on sampling time.

The GCC recommendation on carryover control is shown in Box $\|$.

\section{Future perspective}

The GCC will continue to expand its membership by coordinating its activities with the regional and international meetings held by the pharmaceutical industry. Furthermore, the initiative to accumulate long-term frozen storage stability data in an effort to investigate whether stability is or is not affected by the presence of co-administered compounds is currently evolving. All those who are willing to contribute should contact the GCC for further information [103]. Finally, the next GCC Closed Forums will be scheduled in both North America (GCCNA) and Europe (GCC-EU). Please contact the GCC for the exact date and time of abovementioned meetings, and for all membership information.

\section{Acknowledgments}

The GCC would like to thank Natasha Savoie (Algorithme Pharma) for offcially writing this White Paper with contributions from all authors, and to Fabio Garofolo (Algorithme Pharma) for acting as the coordinator for this article. Additional thanks to all the member representatives who have sent comments and suggestions to complete this report.

Thanks to Eric Woolf(Senior Director, Merck Research Laboratories, USA) and Surendra Bansal (Research Director, Bioanalytical Research \& Development, NonClinical Safety, Hoffmann-La Roche, USA) for choosing the GCC as collaborators for collecting co-administered compound stability data. 


\section{Box 8. The GCC recommendation on rejected evaluations in validation reports.}

- All pertinent evaluations, or at a minimum a reference to where they are presented, should always be included in validation reports, irrespective of the study in which they were performed.

Box 9. The GCC recommendation on stock solutions used for calibrants \& quality control sample preparation.

- After an extensive discussion, it was concluded that the currently accepted procedures of preparing calibrant and quality control samples from a single stock solution are scientifically sound. The GCC is planning to contact the Agency for further clarifications on the scientific reasoning behind this observation.

\section{Box 10. The GCC recommendation on carryover control.}

- For a typical pharmacokinetic study using a method that could not completely eliminate carryover during method development, by injecting samples in order of increasing concentration or in order of sampling timepoints, any carryover present is typically sufficiently under control and the effect on reported concentrations becomes insignificant. In each such batch, the potential significance of carryover should be evaluated using a blank sample injected after the highest standard, at least at the beginning and end of the batch. The area (or height) responses of any peaks present in the blank samples are then compared with those of the mean LLOQ sample response. Peaks $>20 \%$ of the LOQ are an indication that some degree of carryover may be occurring during batch injection. However, this does not necessarily require rejection of the batch or any unknowns within the batch. Using the highest percentage of carryover determined, each sample can then be evaluated taking into consideration the preceding sample. For example, if $25 \%$ carryover (relative to the LOQ) is established, then a carryover factor corresponding to each unknown $(X)$ should be calculated and compared with the response of the following unknown $(X+1)$. If the carryover contribution from $X$ is greater than $5 \%$ of the response of $X+1$, then sample $X+1$ is unreliable and should be repeated. In addition, any sample $X+1$ that follows a sample $X$ with a concentration higher than the standard used to evaluate the impact of carryover should be further investigated, since the impact of above range samples on subsequent injections was not evaluated within the batch in question.

\section{Financial \& competing interests disclosure The authors have no relevant affiliations or financial involvement with any organization or entity with a finan- cial interest in or financial conflict with the subject matter or materials discussed in the manuscript. This includes employment, consultancies, honoraria, stock ownership or options, expert testimony, grants or patents received or pending, or royalties. No writing assistance was utilized in the production of this manuscript.}

\section{Author affiliations}

'Advion BioServices, Ithaca, NY, USA

${ }^{2}$ Agilux Laboratories, Worcester, MA, USA

${ }^{3}$ AIT Bioscience, Indianapolis, IN, USA

${ }^{4}$ Algorithme Pharma Inc., Quebec, Canada

${ }^{5}$ Alphalyse, Odense, Denmark

${ }^{6}$ Alturas Analytics, Moscow, ID, USA

${ }^{7}$ Anapharm Europe, Barcelona, Spain

${ }^{8} \mathrm{BASi}$, West Lafayette, IN, USA

${ }^{9}$ Celerion, Lincoln, NB, USA

${ }^{10}$ Cetero Research, Houston, TX, USA

"Charles River, Senneville, Quebec, Canada

${ }^{12} \mathrm{CIRION}$ BioPharma Research, Laval, Quebec, Canada

${ }^{13}$ Covance, Chantilly, VA, USA

${ }^{14}$ Covance, Madison, WI, USA

${ }^{15}$ Frontage Laboratories, Malvern, PA, USA

${ }^{16} \mathrm{Gen}$-Probe Life Sciences, Manchester, UK

${ }^{17}$ Huntingdon Life Sciences, East Millstone, NJ, USA

${ }^{18}$ ICF, Goiania, Brasil

${ }^{19}$ ICON Development Solutions, Whitesboro, NY, USA

${ }^{20}$ Intertek, El Dorado Hills, CA, USA

${ }^{2}$ JCL Bloassay USA, Hoffman Estates, IL, USA

${ }^{22}$ Lambda Therapeutic Research, Toronto, ON, Canada

${ }^{23}$ MEDTOX Laboratories, St Paul, MN, USA

${ }^{24} \mathrm{MPI}$ Research, Mattawan, MI, USA

${ }^{25}$ Parexel, Bloemfontein, South Africa

${ }^{26}$ Pharma Medica, Toronto, ON, Canada

${ }^{27}$ PharmaNet Canada, Quebec City, Quebec, Canada

${ }^{28} \mathrm{PPD}$, Middleton, WI, USA

${ }^{29}$ PPD, Richmond, VA, USA

${ }^{30}$ PRA International, Lenexa, KS, USA

${ }^{31}$ Pyxant Labs, Colorado Springs, CO, USA

${ }^{32}$ QPS, Groningen, Netherlands

${ }^{33}$ Quotient Bioresearch, Fordham, UK

${ }^{34}$ Smithers Pharma, Wareham, MA, USA

${ }^{35}$ Tandem Labs, West Trenton, NJ, USA

${ }^{36}$ Tandem Labs, Durham, NC, USA

${ }^{37}$ Unilab York Bioanalytical Solutions, York, UK

${ }^{38}$ Warnex Bioanalytical, Laval, Quebec, Canada

${ }^{39}$ WIL Research Company, Ashland, OH, USA

${ }^{40}$ WIL Research Company, Skokie, IL, USA

${ }^{4}$ 'Worldwide Clinical Trials, Austin, TX, USA

${ }^{42}$ XenoBiotic Laboratories, Plainsboro, NJ, USA

notable points in the 2009 draft EMA Guideline and differences with the 2001 FDA Guidance. Bioanalysis 2(5), 929-935 (2010).

4 Savoie N, Garofolo F, van Amsterdam P et al. 2010 White Paper on recent issues in regulated bioanalysis and global harmonization of bioanalytical guidance. Bioanalysis 2(12), 1945-1960 (2010).
5 Shah V, Midha K, Findlay J et al. Bioanalytical method validation - a revisit with a decade of progress. Pharm. Res. 17(12), 1151-1557 (2000)

6 Savoie N, Booth BP, Bradley T et al. 2008 White Paper: the 2nd calibration and Validation Group Workshop on Recent Issues in Good Laboratory Practice Bioanalysis. Bioanalysis 1(1), 19-30 (2009). 
7 Savoie N, Garofolo F, van Amsterdam P et al. 2009 White Paper on recent issues in regulated bioanalysis from the $3^{\text {rd }}$ Calibration and Validation Group Workshop. Bioanalysis 2(1), 53-68 (2010)

8 De Boer T, Wieling J. Incurred sample accuracy assessment: design of experiments based on standard addition. Bioanalysis 3(9), 983-992 (2011)

9 US FDA. Guidance for Industry: Bioanalytical Method Validation. US FDA, Center for Drug Evaluation and Research, MD, USA (2001).
10 Viswanathan CT, Bansal S, Booth B et al. Quantitative bioanalytical methods validation and implementation: best practices for chromatographic and ligand binding assays. Workshop/Conference Report. AAPS J. 9(1), E30-E42 (2007).

11 EMA. Draft Guideline on the Validation of Bioanalytical Methods. EMA, Committee for Proprietary Medicinal Products, London, UK (2009).

\section{- Websites}

101 Future Science, UK. www.future-science.com/action/showMostRe adArticles?journalCode $=$ bio

102 Yau M. Stability of Samples, Standards, QC's, and Reagent: FDA Perspective - Presentation. www.aapspharmaceutica.com/meetings/ files/64/Yau.pdf

103 Global CRO Council. www.canadianlcmsgroup.com/bioanalysis_ gcc_membercompanies.php 